\title{
Intracoronary Injection of Granulocyte Colony- Stimulating Factor Ameliorates the Progression of Left Ventricular Remodeling After Myocardial Ischemia/Reperfusion in Rabbits
}

\author{
Hiroshi Hasegawa, MD; Hiroyuki Takano, MD; Hirokazu Shiraishi, MD; \\ Kazutaka Ueda, MD; Yuriko Niitsuma, MD; \\ Hiroyuki Tadokoro, MD; Issei Komuro, MD
}

\begin{abstract}
Background Although granulocyte colony-stimulating factor (G-CSF) is known to prevent left ventricular (LV) remodeling after acute myocardial infarction (AMI), the best method of administration is unknown.

Methods and Results A rabbit ischemia/reperfusion model was created and G-CSF was administered into the coronary artery immediately after reperfusion. The LV size and contraction were determined by echocardiography, and the extent of infarcted myocardium was measured by Masson-Trichrome staining. The benefits of intracoronary injection of G-CSF on LV remodeling were similar to subcutaneous injection.

Conclusions Direct intracoronary G-CSF injection may become a new therapy for AMI with lower adverse effects. (Circ J 2006; 70: 942-944)
\end{abstract}

Key Words: Granulocyte colony-stimulating factor; Ischemia/reperfusion; Remodeling

W

e and others have reported that granulocyte colony-stimulating factor (G-CSF) prevents left ventricular (LV) remodeling after acute myocardial infarction (AMI) ${ }^{1-5}$ and improves limb ischemia6 We recently demonstrated that it inhibits the progression of $\mathrm{LV}$ remodeling through direct activation of the Jak2/STAT3 pathway, which has anti-apoptotic effects and angiogenic effects, in cardiomyocytes. Because leukocytosis may have a deleterious effect on atherosclerosis and the infarcted myocardium, the most effective method of administrating G-CSF with less activation of bone marrow (BM) and more stimulation on cardiomyocytes is necessary. In the present study, we investigated the effects of direct intracoronary (IC) administration of G-CSF using a rabbit ischemia/reperfusion model.

\section{Animal Models}

The male Japanese white rabbit ischemia/reperfusion model was used ${ }^{5}$ and all protocols were approved by the Institutional Animal Care and Use Committee of Chiba University. Under anesthesia, the carotid artery was cannulated with a 4-F wedge-pressure catheter and the tip of the catheter was placed at the supra-aortic valve. Next, left thoracotomy was performed and the large coronary arterial branch coursing down the middle of the anterolateral surface of the LV was ligated for $30 \mathrm{~min}$. After release of the ligature, the ascending aorta was clamped by the balloon in-

(Received April 5, 2006; revised manuscript received May 12, 2006; accepted May 19, 2006)

Department of Cardiovascular Science and Medicine, Chiba University Graduate School of Medicine, Chiba, Japan

Mailing address: Issei Komuro, MD, Department of Cardiovascular Science and Medicine, Chiba University Graduate School of Medicine (M4), 1-8-1 Inohana, Chuo-ku, Chiba 260-8670, Japan. E-mail: komuro-tky@umin.ac.jp flation of the wedge-pressure catheter and the rabbits were then randomized into 3 groups: (1) control group (CONT): saline injection into the coronary artery $(\mathrm{n}=12) ;(2) \mathrm{G}-\mathrm{CSF}$ IC group (IC): direct IC injection with recombinant human G-CSF (10qu g/kg, rhG-CSF, Kirin Brewery Co Ltd, Tokyo, Japan, $\mathrm{n}=15$ ) for $30 \mathrm{~s}$; and (3) G-CSF SC group; subcutaneous (SC) injection of G-CSF $(10 \mu \mathrm{g} / \mathrm{kg}$ per day $\times 5$ days, $n=10)$.

\section{Measurements}

The number of circulating white blood cells (WBC) was measured at pre-operation and 1, 3, 5, 7 and 14 days after operation. Echocardiography was performed at pre-operation and 14 days after operation. At 14 days, rabbits were killed and the extent of infarcted myocardium in relation to total LV (\%area) was measured by Masson-Trichrome staining.

\section{Statistics}

All measurements are expressed as group mean \pm SEM, and changes in cardiac function and infarct size were analyzed by 2-way repeated-measures ANOVA. Statistical differences were considered significant if the p-value was $<0.05$.

One CONT rabbit died during the study period, but none of the IC or SC rabbits (mortality rate; CONT: $6.6 \%$; IC: 0\%; SC: $0 \%$ ) died. None of the groups showed loss of body weight or other behavioral problems. The number of WBC was more increased in the SC rabbits, and peaked at 7 days compared with the CONT rabbits, which peaked on day 1 (Table 1). In the IC rabbits, the WBC peak was on day 1 and decreased rapidly thereafter.

LV dilatation and dysfunction were less in the IC and SC rabbits than in CONT rabbits (Figs 1A,B). Although the area at risk measured by Evan blue staining was the same 
Table 1 Number of WBCs in Peripheral Blood

\begin{tabular}{|c|c|c|c|c|c|c|}
\hline & \multicolumn{6}{|c|}{ No. WBC $(\not L)$} \\
\hline & Pre & 1 day & 3 days & 5 days & 7 days & 14 days \\
\hline CONT & $7,950 \pm 890$ & $10,500 \pm 1,400$ & $10,000 \pm 1,860$ & $9,800 \pm 1,570$ & $9,720 \pm 1,360$ & $7,010 \pm 390$ \\
\hline$I C$ & $7,720 \pm 360$ & $21,450 \pm 2,860 *$ & $12,910 \pm 2,580$ & $13,010 \pm 2,070$ & $12,180 \pm 1,670$ & $7,200 \pm 400$ \\
\hline$S C$ & $9,850 \pm 1,110$ & $12,500 \pm 1,670$ & $15,900 \pm 2,950 *$ & $23,450 \pm 3,730^{*}$ & $24,520 \pm 3,420 *$ & $10,440 \pm 960 *$ \\
\hline
\end{tabular}

Results are mean $\pm S E M . * p<0.05$ vs CONT.

WBC, white blood cell; CONT, control group; IC, intracoronary; SC, subcutaneous.

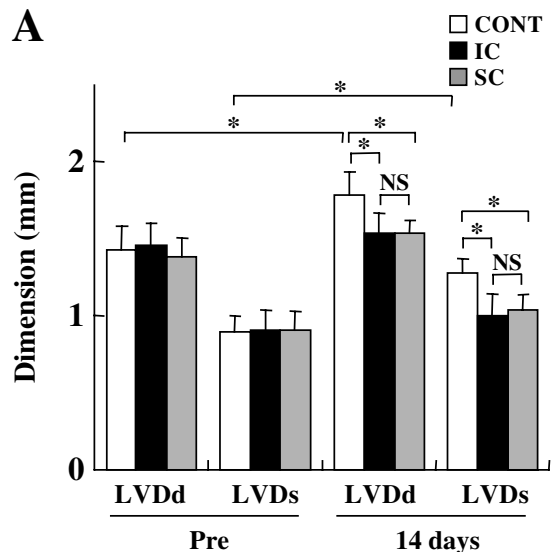

C
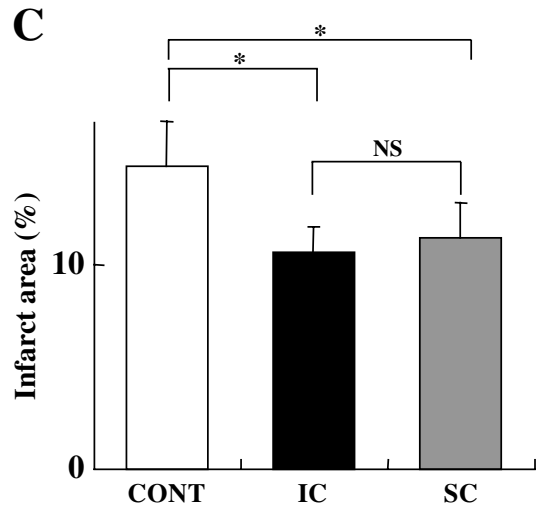

B $\quad$ CCONT
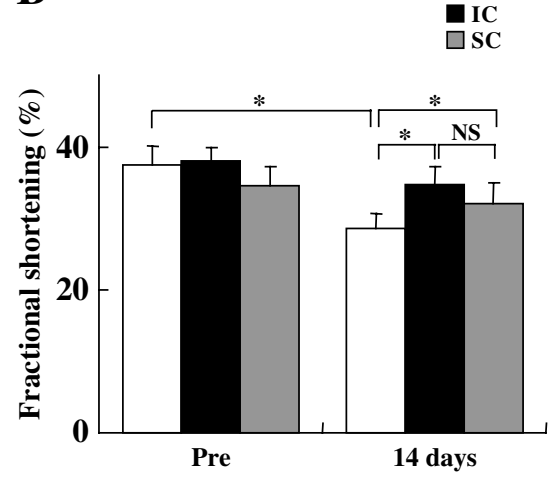

in the 3 groups (CONT: $32.4 \pm 2.3 \%$; IC: $35.7 \pm 2.6 \%$; SC: $33.4 \pm 2.9 \%$ ), the \%area of infarcted myocardium was smaller in the IC and SC hearts compared with the CONT hearts (Fig 1C).

In this study using a rabbit ischemia/reperfusion model, one injection of G-CSF directly into the coronary artery prevented LV remodeling and reduced the infarct size by a degree similar to that with SC injection. Moreover the survival rate was also improved in relation to the inhibition of LV remodeling. Because the level of leucocytosis was less in the IC rabbits compared with the SC rabbits, the leucocytosis-induced systemic side effects are also thought to have been low.

The effects of G-CSF on the AMI-affected heart are mainly anti-apoptotic and angiogenetic through direct activation of the Jak2/STAT3 pathway in cardiomyocytes? In our previous study, the beneficial effects of G-CSF on LV remodeling were dose dependent and significantly reduced by a delayed start of treatment? Because the main effect of G-CSF on the AMI heart is the protection of cardiomyocytes, but not the mobilization of $\mathrm{BM}$ cells, earlier administration and a higher dose of G-CSF will conceiva-
Fig 1. Effect of intracoronary granulocyte colony-stimulating factor (G-CSF) administration on (A) left ventricular end-diastolic diameter (LVDd) and end-systolic diameter (LVDs) and (B) left ventricular fractional shortening determined by echocardiography. (C) Effect of intracoronary G-CSF administration on myocardial infarction size measured by Masson-Trichrome staining. Results are expressed as mean \pm SEM. ${ }^{*} \mathrm{p}<$ 0.05. CONT, control group; IC, intracoronary; SC, subcutaneous. bly protect cardiomyocytes more effectively after AMI. To more effectively protect infarcted myocardium with less systemic side effects, IC administration of G-CSF may be a better method of treating AMI.

Because most AMI patients are treated with percutaneous coronary intervention (PCI), it is very easy and reasonable to inject G-CSF into the coronary artery immediately after PCI. This treatment may become a new therapy for AMI patients and a more detailed study using large animals and a clinical study are awaited.

\section{Acknowledgments}

This work was supported by Health and Labour Sciences Research Grants, Uehara Memorial Foundation, Grant-in-Aid of The Japan Medical Association and Takeda Science Foundation.

\section{References}

1. Ohtsuka M, Takano H, Zou Y, Toko H, Akazawa H, Qin Y, et al. Cytokine therapy prevents left ventricular remodeling and dysfunction after myocardial infarction through neovascularization. FASEB $J$ 2004; 18: 851-853.

2. Harada M, Qin Y, Takano H, Minamino T, Zou Y, Toko H, et al. 
G-CSF prevents cardiac remodeling after myocardial infarction by activating the Jak-Stat pathway in cardiomyocytes. Nat Med 2005; 11: $305-311$

3. Takano H, Qin Y, Hasegawa H, Ueda K, Niitsuma Y, Ohtsuka M, et al. Effects of G-CSF on left ventricular remodeling and heart failure after acute myocardial infarction. J Mol Med 2006; 84: 185-193.

4. Suzuki K, Nagashima K, Arai M, Uno Y, Misao Y, Takemura G, et al. Effect of granulocyte colony-stimulating factor treatment at a low dose but for a long duration in patients with coronary heart disease. Circ J 2006; 70: 430-437.
5. Minatoguchi S, Takemura G, Chen XH, Wang N, Uno Y, Koda M, et al. Acceleration of the healing process and myocardial regeneration may be important as a mechanism of improvement of cardiac function and remodeling by postinfarction granulocyte colony-stimulating factor treatment. Circulation 2004; 109: 2572-2580.

6. Ishida A, Ohya Y, Sakuda H, Ohshiro K, Higashiuesato Y, Nakaema $\mathrm{M}$, et al. Autologous peripheral blood mononuclear cell implantation for patients with peripheral arterial disease improves limb ischemia. Circ J 2005; 69: 1260-1265. 